\title{
Molecular mechanisms of selective autophagy
}

\author{
GM Fimia ${ }^{1}$, G Kroemer ${ }^{2,3,4,5}$ and M Piacentini ${ }^{*, 1,6}$ \\ Cell Death and Differentiation (2013) 20, 1-2; doi:10.1038/cdd.2012.97
}

Autophagy is the catabolic process by which the cell degrades cytoplasmic components within lysosomes. ${ }^{1}$ The balance between the biosynthesis of cellular components and their eventual degradation is of cardinal importance for normal cellular homeostasis and health. ${ }^{2}$ Autophagy has long been thought to be an essential but non-selective bulk degradation pathway. However, recently accumulating evidence has highlighted the selective elimination by autophagy of unwanted components such as aberrant protein aggregates, lipid droplets, dysfunctional organelles and invading pathogens. ${ }^{3}$ Some of the molecular components involved in selective autophagy have been identified, implying that we are beginning to understand how selectivity is achieved in this process. Specific autophagy receptors are responsible for selective autophagy by tethering cargo to the site of autophagosomal engulfment. ${ }^{4}$ In addition, it is becoming clear that post-translational modifications have an important role in ensuring substrate recognition and selectivity in vertebrates. ${ }^{5}$ For example, protein ubiquitination, besides its role in proteasome-mediated degradation, can constitute a modification that targets proteins to turnover by autophagy. ${ }^{5}$ The recognition of ubiquitinated substrates is provided by molecular adaptors including p62, NBR1, NDP52, VCP and optineurin, which bind on one side to ubiquitin and, on the other end, to autophagosome-specific proteins, such as the members of the LC3/GABARAP/Gate16 family. ${ }^{4}$ So far, several autophagy-'specific' receptors and adaptor proteins that regulate the selective degradation of damaged organelles, protein aggregate and pathogens have been identified and partially characterized., ${ }^{4,6}$ However, the physiological roles of selective autophagy are not yet fully understood.

In this thematic issue, five reviews summarize the mechanistic and structural basis of receptor-mediated selective autophagy. Shaid et al. ${ }^{7}$ focus on the role of ubiquitinylation signals and selective autophagy receptors in selecting cargo for autophagic degradation. Suzuki ${ }^{8}$ provides an overview of selective autophagy in yeast, concentrating on Atg11dependent pathways. In particular, Atg11 appears to be important for organelle-selective autophagy, such as mitophagy (selective degradation of mitochondria), pexophagy (selective degradation of peroxisomes) and piecemeal microautophagy of the nucleus. In contrast, ribophagy (ribosomal degradation) could represent a non-selective and Atg-11-independent autophagic pathway. ${ }^{8}$ Ashrafi and Schwarz ${ }^{9}$ describe the state of the art in the process of mitophagy. The selective autophagy of mitochondria is probably the only quality control mechanism that allows for eliminating these organelles upon irreversible damage. Mitophagy also mediates the removal of superfluous mitochondria from differentiating erythrocytes, and contributes to the exclusively maternal inheritance of mitochondrial DNA via the elimination of sperm-derived mitochondria. ${ }^{9}$ Liu and Czaja $^{10}$ analyse 'lipophagy', a recently identified alternative pathway of lipid degradation mediated by lysosomal hydrolases. In this very specific form of selective autophagy, triglycerides and cholesterol contained in lipid droplet are taken up by autophagosomes and delivered to lysosomes for degradation by acidic hydrolases. Intracellular lipids can regulate levels of autophagy by yet unknown mechanisms. Interestingly, impaired lipophagy can promote excessive tissue lipid accumulation such as hepatic steatosis induced by viral infections, ${ }^{11}$ alter hypothalamic neuropeptide release to affect body mass, ${ }^{12}$ block cellular transdifferentiation and sensitize cells to death stimuli. ${ }^{13}$ Finally, Isakson et al. ${ }^{14}$ discuss the current knowledge of the most prominent mammalian adaptor protein identified thus far, autophagylinked FYVE protein (ALFY). ALFY is a large, scaffolding, multidomain protein implicated in the selective degradation of ubiquitinated protein aggregates by autophagy. ${ }^{14}$

The avid reader of this collection of excellent reviews will appreciate that several major problems remain to be solved. Which are the early upstream signals triggering different types of selective autophagy? Do different stress/damage agents activate selective autophagy through common or distinct receptor proteins? How can a balanced crosstalk be achieved between selective autophagy and programmed cell death pathways?

We anticipate that answering these questions will yield clinically useful information because autophagy has an essential role in removing potentially harmful intracellular components. Thus, impaired or disabled autophagy has been linked to many human pathologies including cancer, neurodegeneration, maladaptive immune responses and premature ageing. ${ }^{15}$ In keeping with this, we hope that this CDD review set will contribute to stimulating the interest of the scientific community in this ever more intriguing research field.

\footnotetext{
${ }^{1}$ National Institute for Infectious Diseases IRCCS 'L Spallanzani', Rome, Italy; ${ }^{2}$ INSERM, U848, Institut Gustave Roussy, Villejuif, France; ${ }^{3}$ Metabolomics Platform, Institut Gustave Roussy, Villejuif, France; ${ }^{4}$ Centre de Recherche des Cordeliers, Paris, France; ${ }^{5}$ Pôle de Biologie, Hôpital Européen Georges Pompidou, AP-HP, Paris, France and 'Department of Biology, University of Rome 'Tor Vergata', Rome, Italy

*Corresponding author: M Piacentini, Department of Biology, University of Rome 'Tor Vergata', Via della Ricerva Scientifica 1, Rome 00173 , Italy. Tel: +39 0672594234 ; Fax: +39 067259 4222; E-mail: mauro.piacentini@uniroma2.it
} 
1. Kroemer G, Mariño G, Levine B. Mol Cell 2010; 40: 280-293.

2. Mizushima N, Komatsu M. Cell 2011; 147: 728-741.

3. Hubbard VM et al. Biogerontology 2012; 13: 21-35.

4. Johansen T, Lamark T. Autophagy 2011; 7: 279-296.

5. McEwan DG, Dikic I. Trends Cell Biol 2011; 21: 195-201.

6. Noda NN, Ohsumi Y, Inagaki F. FEBS Lett 2010; 584: 1379-1385.

7. Shaid S et al. Cell Death Differ 2012; 20: 21-30.

8. Suzuki K. Cell Death Differ 2012; 20: 43-48.
9. Ashrafi G, Schwarz TL. Cell Death Differ 2012; 20: 31-42.

10. Liu K, Czaja MJ. Cell Death Differ 2012; 20: 3-11.

11. Vescovo T et al. Gastroenterology 2012; 142: 644-653e3.

12. Kaushik $S$ et al. Cell Metab 2011; 14: 173-183.

13. Fimia GM, Piacentini M. Cell Mol Life Sci 2010; 67: 1581-1588.

14. Isakson $P$, Holland $P$, Simonsen A. Cell Death Differ 2012; 20: 12-20.

15. Levine B, Kroemer G. Cell 2008; 132: 27-42. 